\title{
Displacement of plastic material with an elastic disc
}

\author{
ANDREW PARRY ${ }^{\mathrm{a}}$ \\ Department of Mechanical Technology, Schlumberger Riboud Product Center, 1 rue Henri Becquerel, 92140 Clamart, France
}

Received 1st September 2006, accepted 23 January 2007

\begin{abstract}
The displacement of a plastic fluid by an elastic disc approaching a plane rigid surface oriented perpendicular to the disc axis is computed with a numerical scheme accounting for the coupled deformation of the disc and flow of plastic. The axial force to push the disc is in agreement with lubrication theory approximation. The existence of un-yielded fluid situated at the corners of the fluid region on the centre-line have been predicted, similar to the case observed for rigid disc squeeze flow.
\end{abstract}

Key words: Fluid structure interaction / rubber / plastic / yield stress / Bingham

Résumé - Déplacement d'un matériau plastique avec un disque élastique. Le déplacement d'un fluide plastique par un disque élastique se rapprochant d'une surface rigide plate perpendiculaire à l'axe du disque, est calculé avec un schéma numérique tenant compte à la fois de la déformation du disque et aussi de l'écoulement du fluide. La force axiale résultante est en accord avec la force calculée par une relation analytique pour des écoulements minces. L'existence d'une région de fluide ne dépassant pas la limite d'écoulement, est observée aux coins sur l'axe de symétrie, de la même manière que pour un disque rigide.

Mots clés : Intéraction fluide structure / caoutchouc / plastique / contrainte d'écoulement / Bingham

\section{Nomenclature}

\begin{tabular}{|ll|}
\hline$E$ & Young's modulus in N.m $\mathrm{m}^{-2}$ \\
$h$ & gap between disc and rigid plane in $\mathrm{m}$ \\
$R$ & outer radius of disc in $\mathrm{m}$ \\
$\gamma$ Greek symbols & \\
$\gamma$ & under-relaxation factor for fluid-structure interface coordinates \\
$\lambda$ & magnitude of strain rate tensor in $\mathrm{s}^{-1}$ \\
$\lambda_{0}$ & regularization parameter in $\mathrm{s}^{-1}, \mu_{\mathrm{e}}=\frac{\tau_{\mathrm{y}}}{\lambda}\left[1-e^{-\lambda / \lambda_{O}}\right]$ \\
$\mu_{\mathrm{e}}$ & effective viscosity of mixture in $\mathrm{kg} \cdot \mathrm{m}^{-1} \cdot \mathrm{s}^{-1}$ \\
$\nu$ & Poisson's ratio \\
$\tau_{\mathrm{y}}$ & yield stress in N.m \\
\hline
\end{tabular}

\section{Introduction}

"Squeeze flow" occurs when parallel or nearly parallel surfaces approach each other and compress the fluid between them, which is squeezed out radially [1]. This kind of interaction is of wide reaching engineering interest and is encountered in rheometry, metal working, material forming, contact and sealing research. An example where the compliance of the surfaces are important is the forging

\footnotetext{
a Corresponding author:

aparry@clamart.oilfield.slb.com
}

of a metal part in a die where there is fluid-structure interaction between the deforming part and the lubricant. Another example, from the oilfield industry, is the sealing or isolation of different parts of the well with inflatable packers. The interaction of these structures with the visco-plastic cake on the well bore and the resulting sealing efficiency are important issues. A multi-dimensional calculation method for prediction of filter cake growth and deformation is given in [2].

Recent investigations into squeeze flows of viscoplastic fluids between approaching rigid discs have been 

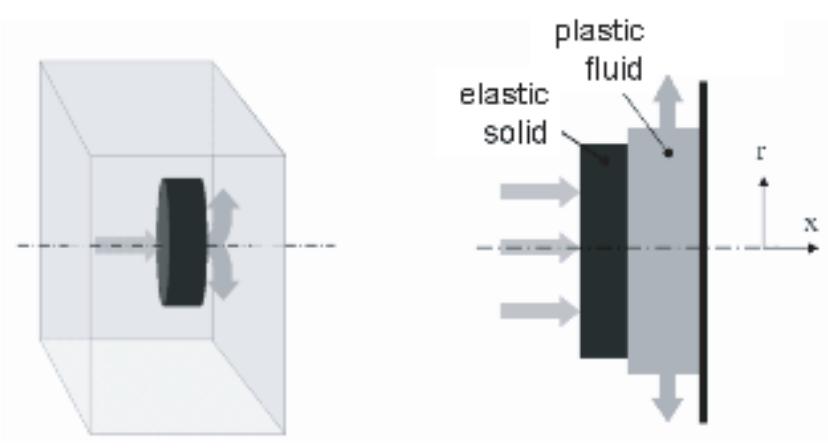

Fig. 1. Schematic of disc approaching rigid surface.

reported by, for example, Sherwood \& Durban [3] and by Matsoukas \& Mitsoulis [4]. These works provide analytical and empirical equations describing the force required to compress the fluid between the discs.

In this paper, the effect of compliance of the discs will be studied by computing the squeeze flow of a plastic fluid caused by an elastic disc approaching slowly a rigid plane surface, see Figure 1. The fluid and disc are treated as incompressible.

After describing the algorithm for the coupling of the structure deformation with the fluid flow results of a calculation of a thick rubber disc approaching a plane rigid surface, displacing a plastic fluid, are presented. The adequacy of lubrication theory approximation for the fluid forces are addressed.

\section{Implicit fluid-structure coupling algorithm}

\subsection{Fluid flow and structural dynamics solvers}

The fluid dynamics code used for this work, CAFFA (Computer Aided Fluid Flow Analysis) [5], is based on the finite volume approach with arbitrary LagrangianEulerian moving mesh formulation [6]. The dependent variables were the velocity components and pressure. The formulation is non-orthogonal, cell-centered with collocated variables. The structural dynamics code used for this work, DLEARN [7], uses the finite element method with small displacement Lagrangian formulation. Here the dependent variables were displacements. Since a nearly incompressible solid will be considered, the $\bar{B}$ approach [7] for nearly incompressible materials is used along with reduced integration. The finite-elements and finite-volumes meshes are comprised of quadrilaterals. The meshes were coincident at the fluid-structure interface. The fluid flow equations are solved using implicit time integration whereas the solid is at all instants assumed to be in static equilibrium, since in the present work we focus on slow events.

\subsection{Coupling method}

Figure 2 shows a flow chart for the coupling procedure during a time step. Several fluid structure interaction (FSI) iterations are carried out during the time step.

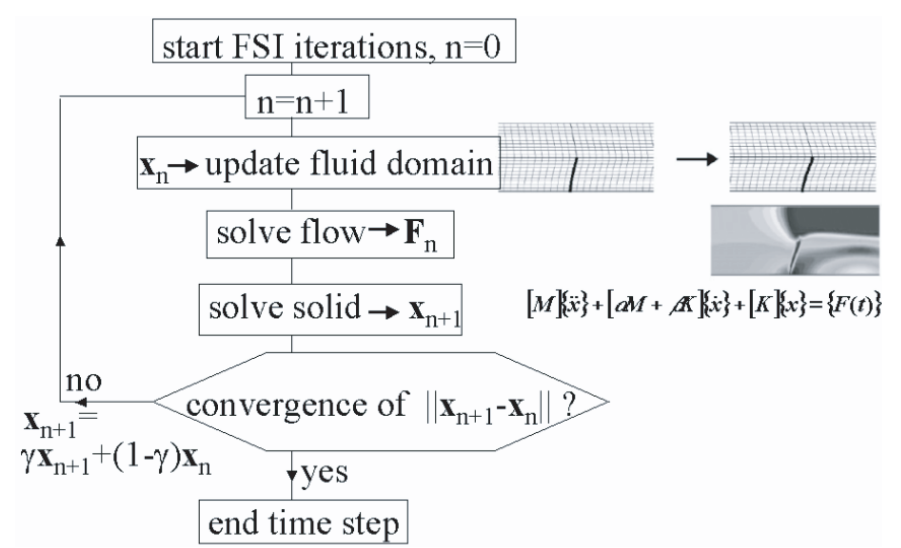

Fig. 2. Flow and structure coupling scheme during one time step.

At the end of the time step, the forces at the domain interfaces are in equilibrium; that is the fluid forces acting on the solid oppose exactly the solid forces acting on the fluid. The fluid flow solver calculates the forces at the shared interfaces; these forces are used as structure boundary conditions. The structure solver determines the interface coordinates; these coordinates are then subsequently used to update the fluid domain geometry. Underrelaxation is applied on the interface coordinates updates. Typically, for stiff problems several tens or hundreds of fluid structure interaction iterations may be required. The convergence criterion for the time step is that the norm of changes in interface coordinates be less than a predetermined tolerance. For the work in this paper, the value set for the interface coordinate under-relaxation factor $\gamma$ was 0.35 and up to $20 \mathrm{FSI}$ iterations were required to obtain convergence to machine accuracy.

The algorithm is an extension of the algorithm developed for rigid structure-fluid interaction developed in a separate paper [8].

\section{Simulation}

\subsection{Problem description}

In this section is described the axisymmetric problem shown in Figure 1.

The fluid is considered as an incompressible plastic with von Mises yield criterion and associated flow rule, with yield stress, $\tau_{\mathrm{y}}=51000 \mathrm{~N} . \mathrm{m}^{-2}$. The effective viscosity, $\mu_{\mathrm{e}}$, is modeled by the Papanastasiou model [9] for a plastic fluid

$$
\mu_{\mathrm{e}}=\frac{\tau_{\mathrm{y}}}{\lambda}\left[1-e^{\frac{\lambda}{\lambda_{0}}}\right]
$$

$\lambda$ is the magnitude of the strain rate tensor $\left(\lambda=\sqrt{\frac{1}{2} I I_{\lambda}}\right.$, where $I I_{\lambda}$ is the second invariant of strain rate) and $\lambda_{\mathrm{o}}$ is an arbitrarily small regularization parameter which avoids convergence problems related to very high viscosity as the strain rate tends to zero. For the velocities in this work, a value of $\lambda_{\circ}$ of $0.01 \mathrm{~s}^{-1}$ was determined to 


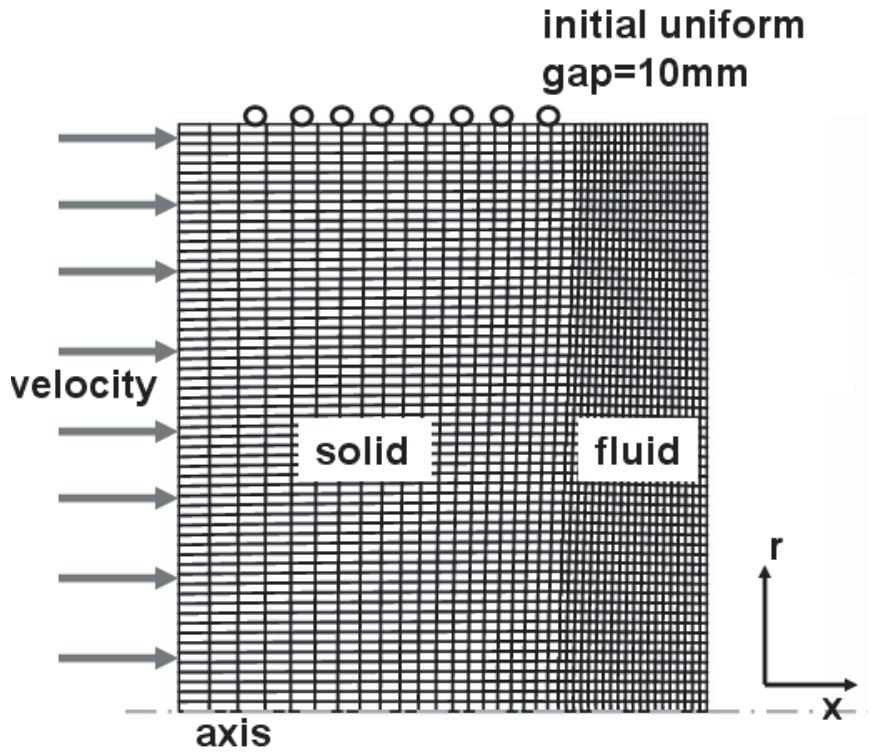

Fig. 3. Boundary conditions and finite-element/finite-volume meshes.

be sufficiently small to provide the true yield stress. During the iterative fluid solving step of each FSI iteration, under-relaxation factors for the fluid velocity components, pressure and effective viscosity were $0.3,0.3,0.05$ and 0.05 respectively. Such low values were required in this case of high yield stress fluid and necessitated a relatively high number of iterations to obtain fully converged fluid variables.

The disc was of diameter $60 \mathrm{~mm}$ and thickness $20 \mathrm{~mm}$, with isotropic properties defined by Young's modulus, $E=5 \times 10^{6}{\mathrm{~N} . \mathrm{m}^{-2}}^{-2}$ and Poisson's ratio, $\nu=0.4999$.

Figure 3 shows the boundary conditions applied. The back of the disc is rigid with uniform axial velocity of initially $0.005 \mathrm{~m} . \mathrm{s}^{-1}$. The velocity imposed at the disc back face is modified to respect a constant ratio of velocity to minimum gap as the disc approaches the rigid surface. The initial condition is zero stress field and uniform gap of $0.01 \mathrm{~m}$. The time step chosen was $0.1 \mathrm{~s}$.

The computation was stopped just before contact occurred between the front of the deformable disc and the rigid wall.

At the fluid flow outlet, situated at the outer radius of the fluid domain, a constant pressure boundary condition was applied. All pressures values quoted later will be relative to this constant stress.

\subsection{Analytical relation for axial force on disc}

The axial force on a rigid disc with radius, $R$, displacing a perfectly plastic fluid with a gap between the disc and rigid surface of $h(r)$, can be approximated by

$$
F=2 \pi \int_{0}^{R}\left[-p(r)+\sigma_{x}(r)\right] r \mathrm{~d} r
$$

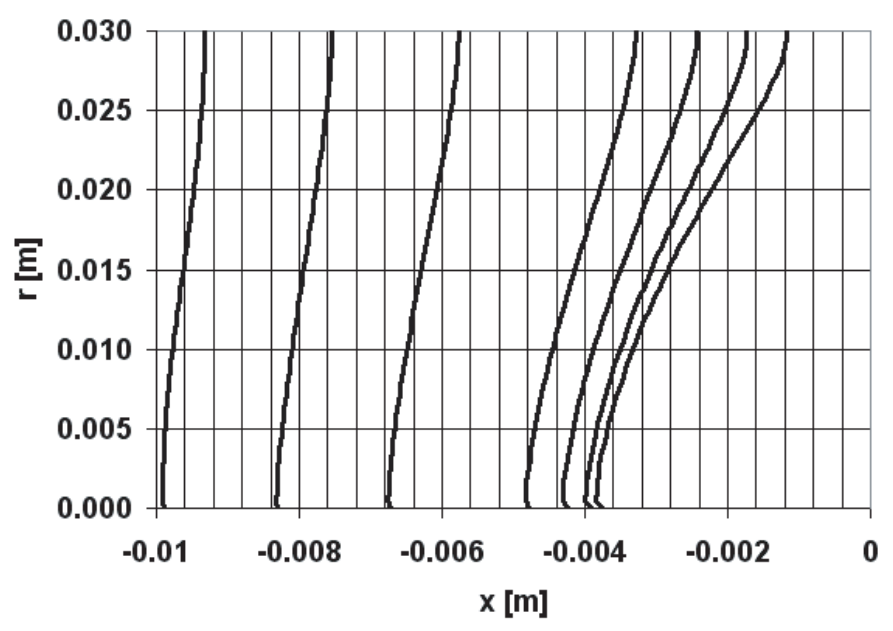

Fig. 4. Fluid-structure interface evolution during approach of flexible disc.

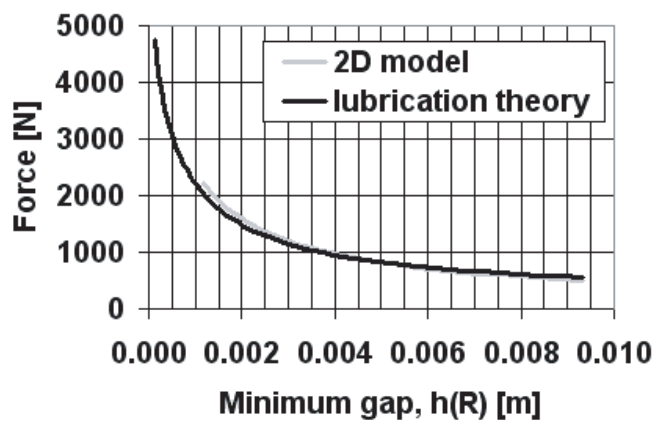

Fig. 5. Axial force evolution during approach of flexible disc.

where $\sigma_{x}=3^{1 / 2} \tau_{\mathrm{y}}$ and pressure increment $\delta p$ between two radial positions with mean gap $h$ is expressed by lubrication theory as $\delta p=\frac{2 \tau_{\mathrm{y}} \delta r}{h}$. The pressure at the outer radius being zero, permits the calculation of $p(r)$ starting at the outer radius and summing increments towards the inner radius.

\subsection{Simulation of disc approaching rigid surface}

The fluid-structure interaction problem was simulated using the method described in 2. Figure 4 shows the evolution of the fluid-structure interface. The minimum gap occurs at the outer radius. A volume of fluid becomes trapped due to the closing of the flow outlet as the disc approaches the rigid surface.

Figure 5 shows the evolution of axial force on the solid disc as a function of minimum gap. There is good comparison between the $2 \mathrm{D}$ axi-symmetric numerical simulation result and that where the fluid forces come from the analytical expression for force given in 3.2. With the analytical fluid force approach, it is possible to continue the calculation further towards the rigid surface as this avoids the numerical difficulties associated with the nonlinear 2D fluid calculation.

Figure 6 shows solid and fluid total axial stress at $2 \mathrm{~s}$ as well as velocity vectors. Note the same levels of solid and 


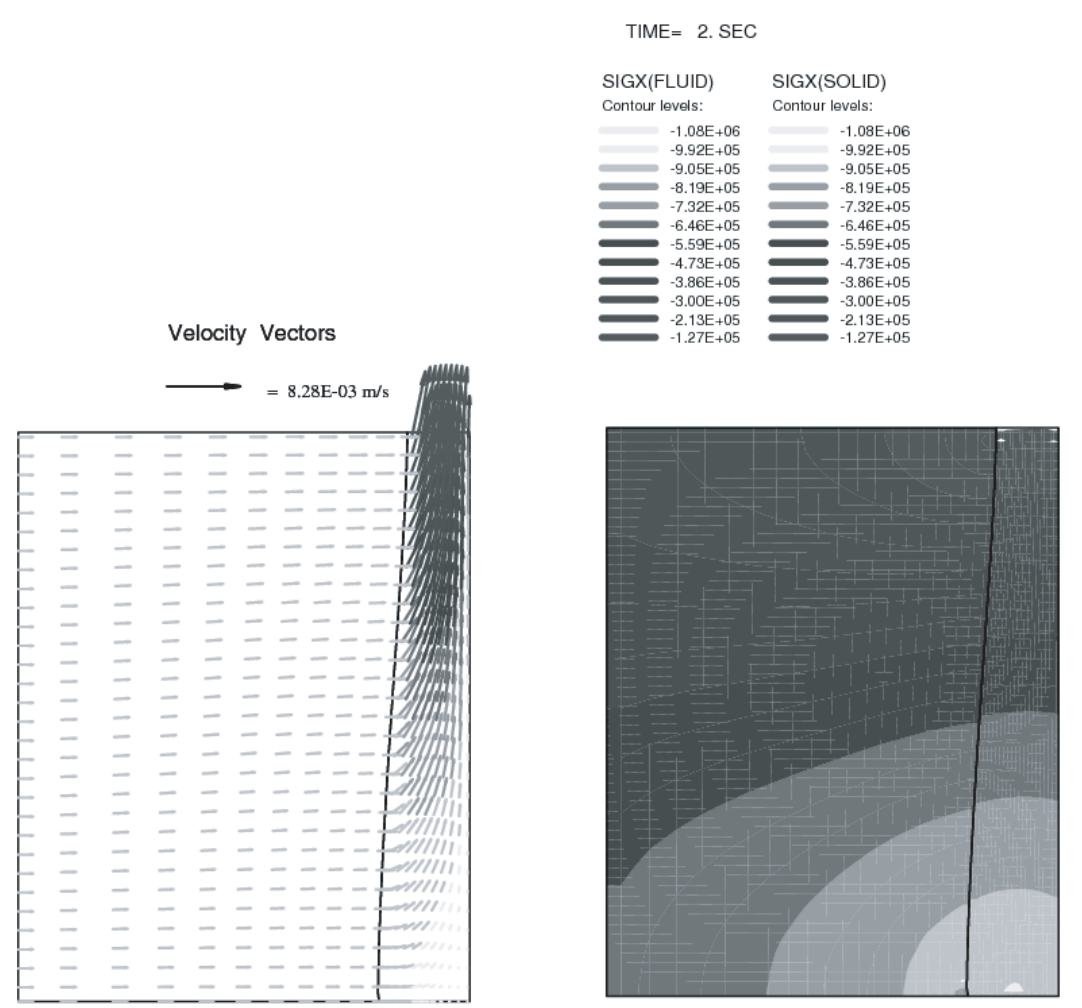

Fig. 6. Fluid and solid velocity vectors and total axial stress $\left[\mathrm{N} . \mathrm{m}^{-2}\right]$ at time $2 \mathrm{~s}$.

fluid axial stress at the fluid-solid interface. The velocity vectors show the continuity of velocity at the interface.

Figure 7 shows the solid pressure and fluid effective viscosity at $3 \mathrm{~s}$. The regions of high fluid viscosity at the corners on the centre-line correspond to un-yielded material. These regions also exist for rigid parallel plate squeeze flow of visco-plastic material [4].

\section{Discussion}

The axi-symmetric problem of a thick elastic disc approaching a plane rigid surface, displacing a plastic fluid, has been studied using an implicit fluid-structure interaction scheme accounting for the deformation of the structure and the fluid flow. Comparison of the squeeze force with an algorithm using an analytical expression for fluid forces validates the algorithm. The existence of un-yielded fluid situated at the corners of the fluid region on the centre-line have been predicted, similar to the case observed for rigid disc squeeze flow. A future work will be to extend the algorithm to the case of non-homogeneous fluids, for example filter cakes [2], and to the case of complex constitutive laws for the structure.

Acknowledgements. I thank Dr. Philippe Hocquet, General Manager, Schlumberger Vector SA, Zone Industrielle, 8 route de Vauchelles, 80142 Abbeville, France, for his advice on the method and for fruitful discussions and aid during the preparation of the paper.
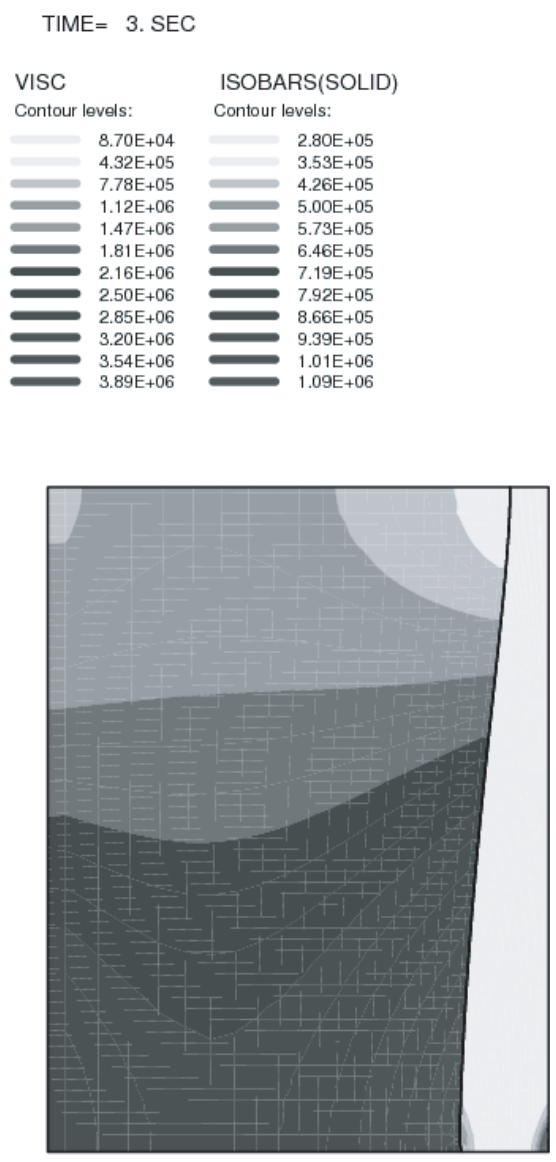

Fig. 7. Fluid effective viscosity $\left[\mathrm{kg} \cdot \mathrm{m}^{-1} \cdot \mathrm{s}^{-1}\right]$ and solid pressure $\left[\mathrm{N} . \mathrm{m}^{-2}\right]$ at time $3 \mathrm{~s}$. 


\section{References}

[1] J. Engmann, C. Servais, A.S. Burbridge, Squeeze flow theory and applications to rheometry: A review, J. NonNewtonian Fluid Mech. 132 (2005) 1-27

[2] A. Parry, Numerical prediction method for growth and deformation of filter cakes, J. Fluids Eng. 128 (2006) 12591265

[3] J.D. Sherwood, D. Durban, Squeeze flow of a power-law viscoplastic solid, J. Non-Newtonian Fluid Mech. 62 (1996) $35-54$

[4] A. Matsoukas, E. Mitsoulis, Geometry effects in squeeze flow of Bingham plastics, J. Non-Newtonian Fluid Mech. 109 (2003) 231-240
[5] J.H. Ferziger, M. Peric, Computational methods for fluid dynamics, Springer, 3rd Ed., ISBN 3-540-42074-6, 2002

[6] I. Demirdzic, M. Peric, Finite volume method for prediction of fluid flow in arbitrary shaped domains with moving boundaries, Int. J. Num. Meth. Fluids 10 (1990) 771-790

[7] T.J.R. Hughes, The finite element method: linear static and dynamic finite element analysis, Dover Publications, ISBN 0-486-41181-8, 2000

[8] D. Abouri, A. Parry, A. Hamdouni, A stable fluid rigid body interaction algorithm: Application to industrial problems, J. Pressure Vessel Technology 128 (2006) 516-524

[9] T.C. Papanastasiou, Flows of materials with yield, J. Rheol. 31 (1987) 385-404 